\title{
Correction to: Assessment of Structural and Functional Comparability of Biosimilar Products: Trastuzumab as a Case Study
}

\author{
Srishti Joshi ${ }^{1}$ Anurag S. Rathore ${ }^{1}[$
}

Published online: 4 September 2020

C) Springer Nature Switzerland AG 2020

Correction to: BioDrugs (2020) 34:209-223 https://doi.org/10.1007/s40259-020-00404-3

Page 215, Section "3.1.2 Primary Structure: Peptide Mapping, Posttranslational Modification, and Degradation Product Identification", left column, second paragraph:

The sentence, which previously read:

"No significant difference (Student's $t$ test) was observed in any of the biosimilars relative to the originator for oxidation at M255 and M431 (Fig. 3c; Table 5 in the ESM)."

Should read:

"No significant difference (Student's t test) was observed in any of the biosimilars relative to the originator for oxidation at M255 and M431 (Fig. 3a; Table 5 in the ESM).”

Page 217, legend to Figure 3 "Glycoform analysis and posttranslational modifications of trastuzumab biosimilars and originator using liquid chromatography mass spectrometry":

The legend, which previously read:

"Relative quantification of a glycoforms for intact trastuzumab samples, b glycoforms for reduced trastuzumab samples, and $\mathbf{c}$ predicted posttranslational modifications for

The original article can be found online at https://doi.org/10.1007/ s40259-020-00404-3.

Anurag S. Rathore

Asrathore@biotechcmz.com

1 Department of Chemical Engineering, Indian Institute of Technology, Delhi, Hauz Khas, New Delhi 110016, India trastuzumab originator and biosimilars, all normalized to the originator."

Should read:

"Relative quantification of a predicted posttranslational modifications for trastuzumab originator and biosimilars, b glycoforms for intact trastuzumab samples, and c glycoforms for reduced trastuzumab samples, all normalized to the originator." 